\title{
Nosocomial bloodstream infection and the emerging carbapenem-resistant pathogen Ralstonia insidiosa
}

\author{
Qingqing Fang ${ }^{1,2}$, Yu Feng ${ }^{1,2}$, Ping Feng ${ }^{1}$, Xiaohui Wang ${ }^{1,2^{*}}$ (D) and Zhiyong Zong ${ }^{1,2,3}$
}

\begin{abstract}
Background: Ralstonia picketti, Ralstonia mannitolilytica, and Ralstonia insidiosa have recently been regarded as emerging pathogens of infectious diseases, in particular as the pathogens responsible for nosocomial infection in immunocompromised patients. R. insidiosa differs from $R$. picketti and $R$. mannitolilytica, and its related infections are rarely reported.

Methods: Clinical data from two nosocomial bloodstream infection cases were extracted and analyzed. The causable isolates were identified by the VITEK 2 Compact system, matrix assisted laser desorption ionization-time of flight mass spectrometry (MALDI-TOF MS), and molecular identification methods using PCR with universal and species-specific primers. Antimicrobial susceptibility testing was performed using the broth microdilution method. Both of the isolates were subjected to whole genome sequencing using a HiSeq X10 Sequencer. Antimicrobial resistance genes, virulence factors, and plasmid replicons were identified from assembled genomes. A real-time RT-PCR experiment and a cloning experiment were conducted to explore the related class D $\beta$-lactamase-encoding genes.
\end{abstract}

Results: Both patients recovered under therapy with antibiotics. Isolates were initially misidentified as $R$. mannitolilytica by the VITEK 2 Compact system rather than $R$. insidiosa, as identified by both MALDI-TOF MS and 16S rRNA gene sequencing. Both isolates were resistant to aminoglycosides, $\beta$-lactams, and polymyxin B. One isolate harboring bla $a_{\mathrm{OXA}}$ 570 was resistant to carbapenems. The whole genome sequencing data confirmed species identification based on average nucleotide identity (ANI) and revealed two variants of class D $\beta$-lactamase-encoding gene bla OXA (bla OXA-573 and bla $\left.a_{\mathrm{OXA}-574}\right)$. The real-time RT-PCR experiment showed no difference in gene expression between bla $a_{\mathrm{OXA}-570}$ and bla $a_{\text {OXA-573 }}$ in our strains. The cloning experiment showed that variant OXA-573 had no carbapenem hydrolase activity.

Conclusions: We described two cases of nosocomial bloodstream infection caused by $R$. insidiosa strains. MALDI-TOF MS was cost-effective for rapid species identification. Clinicians should be aware that $R$. insidiosa can be resistant to commonly used antibiotics, even carbapenems.

Keywords: Ralstonia insidiosa, Carbapenem-resistant, Bloodstream infections, Nosocomial infection, Whole genome sequencing

\footnotetext{
* Correspondence: wang_xiaohui@scu.edu.cn

${ }^{1}$ Center of Infectious Diseases, West China Hospital, Sichuan University,

Chengdu, China

${ }^{2}$ Division of Infectious Diseases, State Key Laboratory of Biotherapy, Chengdu,

China

Full list of author information is available at the end of the article
}

(c) The Author(s). 2019 Open Access This article is distributed under the terms of the Creative Commons Attribution 4.0 International License (http://creativecommons.org/licenses/by/4.0/), which permits unrestricted use, distribution, and reproduction in any medium, provided you give appropriate credit to the original author(s) and the source, provide a link to the Creative Commons license, and indicate if changes were made. The Creative Commons Public Domain Dedication waiver (http://creativecommons.org/publicdomain/zero/1.0/) applies to the data made available in this article, unless otherwise stated. 


\section{Background}

The six effectively published species defining type strains of the genus Ralstonia (Ralstonia insidiosa, Ralstonia mannitolilytica, Ralstonia picketti, Ralstonia pseudosolanacearum, Ralstonia solanacearum, and Ralstonia syzygii) are aerobic gram-negative, non-fermentative, and rod-shaped bacteria. The bacteria are usually isolated from plants and soils. $R$. picketti, $R$. mannitolilytica and $R$. insidiosa have been recently regarded as pathogens of infectious diseases, especially as causable agents of nosocomial infection related to immunocompromised patients [1]. Contaminated solutions or water are believed to be the sources of Ralstonia nosocomial infections [1, 2]. The previous studies showed that most cases of infection were caused by $R$. picketti, with a few caused by $R$. mannitolilytica. The pathogens may cause bloodstream infection, pneumonia, peritonitis, meningitis, endocarditis, spinal osteitis, osteomyelitis, septic arthritis, and prostatitis. $R$. insidiosa was proposed as a new species in 2003 [3]. Bloodstream infection-related cases are extremely rare; none have been reported from Asia. In addition, further information concerning $R$. insidiosa, such as antimicrobial susceptibility, genomic profile and epidemiology, is also limited. Such information is clinically relevant, as $R$. insidiosa may have been underdiagnosed for the past several decades due to its low incidence. The bacterium could also easily be overlooked by researchers. Hence, in this study we described two cases of nosocomial bloodstream infection caused by $R$. insidiosa strains, including a carbapenems-resistant type.

\section{Methods}

\section{Data collection}

Two cases occurred among inpatients in an orthopedics ward at a 5000-bed tertiary hospital in western China. The clinical data were retrieved from medical records and included demographics, symptoms, physical examination findings, laboratory results, comorbidities, treatments, and outcomes. Three physicians independently reviewed the data and defined both cases as nosocomial bloodstream infections.

\section{Isolation and identification}

During hospitalization, when fever was noticed, two sets of peripheral blood cultures were collected in both aerobic and anaerobic bottles (BioMérieux, Marcy-l'Étoile, France). Secondary samples cultured in brucella agar plates supplemented with defibrinated sheep blood (5\% $v / v)$ were incubated aerobically at $35^{\circ} \mathrm{C}$ for up to $48 \mathrm{~h}$. Isolates were initially identified with both the VITEK 2 Compact system (BioMérieux, Marcy-l'Étoile, France) and matrix-assisted laser desorption ionization-time of flight mass spectrometry (MALDI-TOF MS) (IVD MALDI Biotyper system, Microflex LT/SH, Bruker,
Billerica, MA). For automatic biochemical identification, VITEK $^{\oplus} 2$ GN card and VITEK ${ }^{\oplus} 2$ systems software version 7.01 were used. For mass spectrometry identification, Microflex LT was used to collect spectra, and the software FlexControl 3.4 (Bruker Daltonik) was used to analyze data.

Further species identification was established using partial 16S rRNA gene sequencing with universal primers 27F/1492R and species-specific primers Rp-F1/ R38R1 [4]. Nucleotides of amplicons were compared against those of $R$. insidiosa strain ATCC 49129 and FC1138 using the BLASTn algorithm (http://blast.ncbi. nlm.nih.gov/Blast.cgi).

\section{Antimicrobial susceptibility testing}

The minimum inhibitory concentrations (MICs) of 18 antimicrobial agents, which were automatically performed by VITEK 2 Compact system, were manually confirmed using the broth microdilution method (Table 1). Pseudomonas aeruginosa ATCC 27853 and Escherichia coli ATCC 25922 were tested alongside as quality control strains. Interpretation of MIC results was made following the recommendations of breakpoints for Pseudomonas spp., Burkholderia cepacia, and Acinetobacter spp. from the Clinical and Laboratory Standards Institute (CLSI) [5].

\section{Whole genome sequencing, species confirmation and phylogenetics}

Isolates were cultured overnight in $50 \mathrm{~mL}$ BHI broth. Genomic DNA was extracted using a DNeasy Blood \& Tissue Kit (QIAGEN, Hilden, Germany). Quantification and quality assays were performed using a Qubit 2.0 fluorometer and a NanoDrop 2000/2000c spectrophotometer. The extracted DNA was sheared into $350 \mathrm{bp}$ ultrasonically prior to a $150 \mathrm{bp}$ paired-end library construction and then sequenced using a HiSeq X10 Sequencer (Illumina, San Diego, CA, USA). The raw sequencing reads of isolates WCHRI065162 and WCHRI065437 were trimmed using Trimmomatic v0.38 [6] prior to being assembled into draft genomes using SPAdes v3.12.0 [7] under the careful mode. The sequences were deposited into GenBank with assigned accession numbers PKPC00000000 and PKPB00000000, respectively. Antimicrobial resistance genes, virulence factors, and plasmid replicons were identified using whole genome data by the program ABRicate v0.8 (https:/github.com/tseemann/abricate). Genome-based species identification was performed by comparing the average nucleotide identity (ANI) against each existing species of genus Ralstonia using the web program Jspecies (http://imedea.uib-csic.es/jspecies/). The relatedness between strains was described by the number of single nucleotide polymorphisms (SNPs) in the core genome 
Table 1 Resistance patterns of two $R$. insidiosa isolates

\begin{tabular}{lll}
\hline Antibiotics & $\begin{array}{l}\text { WCHRI065437 } \\
(\mu \mathrm{g} / \mathrm{mL})\end{array}$ & $\begin{array}{l}\text { WCHRI065162 } \\
(\mu \mathrm{g} / \mathrm{mL})\end{array}$ \\
\hline Amikacin & $64(\mathrm{R})$ & $128(\mathrm{R})$ \\
Amoxicillin/Clavulanate & $32(\mathrm{R})$ & $32(\mathrm{R})$ \\
Ampicillin & $32(\mathrm{R})$ & $32(\mathrm{R})$ \\
Aztreonam & $256(\mathrm{R})$ & $512(\mathrm{R})$ \\
Cefepime & $2(\mathrm{~S})$ & $16(\mathrm{l})$ \\
Ceftazidime & $8(\mathrm{~S})$ & $16(\mathrm{I})$ \\
Ceftriaxone & $0.5(\mathrm{~S})$ & $0.5(\mathrm{~S})$ \\
Ciprofloxacin & $<0.125(\mathrm{~S})$ & $<0.125(\mathrm{~S})$ \\
Gentamicin & $32(\mathrm{R})$ & $16(\mathrm{R})$ \\
Imipenem & $1(\mathrm{~S})$ & $8(\mathrm{R})$ \\
Levofloxacin & $0.125(\mathrm{~S})$ & $0.25(\mathrm{~S})$ \\
Meropenem & $1(\mathrm{~S})$ & $8(\mathrm{R})$ \\
Nitrofurantoin & $512(\mathrm{R})$ & $512(\mathrm{R})$ \\
Piperacillin/Tazobactam & $<4 / 4(\mathrm{~S})$ & $<4 / 4(\mathrm{~S})$ \\
Polymyxin B & $>512(\mathrm{R})$ & $>512(\mathrm{R})$ \\
Tigecycline & $0.5(\mathrm{~S})$ & $1(\mathrm{~S})$ \\
Tobramycin & $32(\mathrm{R})$ & $32(\mathrm{R})$ \\
Trimethoprim/Sulfamethoxazole & $<0.25 / 4.75(\mathrm{~S})$ & $<0.25 / 4.75(\mathrm{~S})$ \\
\hline R. & &
\end{tabular}

$\mathrm{R}$, resistant; S, susceptible; I, intermediate

identified using Roary v3.11.2 [8] with default settings. The core SNP-based phylogenetic tree was inferred using RAxML v 8.2.12 [9] under the GTRGAMMA model with 1000 bootstrap resamples. Comparative analysis was performed among genomes of our isolates and published $R$. insidiosa strains ATCC 49129 and FC1138. Using ATCC 49129 as the reference, a circular map was generated by the BLAST Ring Image Generator (BRIG) [10].

Real-time RT-PCR and cloning experiments to explore the related class $D \beta$-lactamase-encoding genes

A real-time RT-PCR experiment was conducted to compare gene expression of bla $a_{\text {OXA-60-like }}$ at the mRNA level in our two strains. Total RNA was obtained using an RNAprep pure Cell/ Bacteria Kit (TIANGEN BIOTECH, Beijing, China). The concentrations and quality of RNA were determined by measuring the absorbance at 260 nm. A PrimeScript ${ }^{\text {tm }}$ RT reagent Kit with gDNA Eraser (Takara Bio, Beijing, China) was used for reverse transcriptase PCR (RT-PCR). Real-time RT-PCR was performed using primers described in Table 2 and the house-keeping gene rpoB as the internal control. Amplification was carried out in a $25 \mu \mathrm{L}$-final-volume containing $12.5 \mu \mathrm{L}$ TB Green Premix Ex taqII mix, $1 \mu \mathrm{L}$ primers (5 nM each), $8.5 \mu \mathrm{L} \mathrm{H}_{2} \mathrm{O}$, and $2 \mu \mathrm{L} \mathrm{cDNA}$. The running protocol was denaturation at $95^{\circ} \mathrm{C}$ for $90 \mathrm{~s}$, followed by 40 cycles of denaturation at $95^{\circ} \mathrm{C}$ for $10 \mathrm{~s}$, annealing at $52{ }^{\circ} \mathrm{C}$ for $10 \mathrm{~s}$, and elongation at $72{ }^{\circ} \mathrm{C}$ for 30 s. The
Table 2 Primers used for amplification and sequencing

\begin{tabular}{ll}
\hline Primer & Sequence $\left(5^{\prime} \rightarrow 3^{\prime}\right)$ \\
\hline OXA569-up-EcoRl & AACGAATCATGATGAAACTCCGCCACGC \\
OXA569-down-Sacl & AACGAGCTCAAATCAGTGACTCGCAAGGGCCA \\
OXA570-up-EcoRl & AACGAATCATGTTGCTCGCTGGTCAAA \\
OXA570-down-Sacl & AACGAGCTCAAACTAGGGTGTGGCCACA \\
OXA573-up-EcoRl & AACGAATCATGTTCCCTCGCTGGTCAAA \\
OXA573-down-Sacl & AAAGAGCTCAAACTAGGGTGTGGCCAC \\
OXA574-up-EcoRl & AACGAATTCATGATGAAACTCCGCCACGC \\
OXA574-down-Xhol & AACCTGCAGAAATCAGTGGCTCGCAAGGGC \\
M13 R & GTAAACGACGGCCAGT \\
M13F & CAGGAAACAGCTATGACC \\
OXA570-RT-F & TCAAGCGCACGCCGAGTTGA \\
OXA570-RT-R & GGCACCCGTATCGAAGGC \\
OXA573-RT-F & ATGACCTCAAGCGCGTGTC \\
OXA573-RT-R & GCACCCGTATCGAAGGC \\
RpoB-R.in-F & GTCCATCAGGTTCCCTTCC \\
RpoB-R.in-R & GGACAGGTGATACGACACGA \\
\hline
\end{tabular}

${ }^{a}$ Restriction sites located in primers are underlined

$2^{-\Delta \Delta C}$ Thethod [11] was used to quantify the exact ratio of the genes.

A cloning experiment was conducted to assess functionality of related class D $\beta$-lactamase-encoding genes in our two strains. The host cell was Escherichia coli DH10B (CUNMAI Biotechnologies, Shanghai, China). The cloning and expression vector was a chloramphenicol-resistant plasmid pBC-SK (NovoPro Biotechnology, Shanghai, China). According to a standard directional cloning molecular technique [12], the $b l a_{\text {OXA-60-like }}$ genes $\left(b l a_{\text {OXA-570 }}, b l a_{\text {OXA-573 }}\right)$ and the $b l a_{\text {OXA-22-like }}$ genes $\left(b l a_{\text {OXA-569 }}, b l a_{\text {OXA-574 }}\right)$ found in our strains were amplified using self-designed primers (Table 2), followed by a restriction digestion using selected endonucleases. Recombinant plasmids were transformed into competent $E$. coli $\mathrm{DH} 10 \mathrm{~B}$ cells after ligation by the heat-shock method. The transformed recipient cells were challenged on the chloramphenicol $(40 \mu \mathrm{g} / \mathrm{mL})$ Luria-Bertani (LB) plates for $16 \mathrm{~h}$ at $37^{\circ} \mathrm{C}$. Selected colonies were amplified using the universal primer M13 and sequenced to ensure that no mutation occurred. All E. coli DH10B strains harboring recombinant plasmids expressing OXA-570 (pBC1), OXA-573 (pBC2), OXA-569 (pBC3) and OXA-574 (pBC4) were tested for antimicrobial susceptibility to amoxicillin, ampicillin, cefepime, cefoxitin, ceftazidime, imipenem and meropenem using the broth microdilution method mentioned above. An $E$. coli DH10B strain without the transformed plasmid was included as a reference. 


\section{Results}

\section{Clinical information of case 1}

A 43-year-old male was diagnosed with open multiple fractures and neurovascular injury in the left tibia, fibula, and radius. The patient immediately received debridement on the left leg and left hand. The patient underwent subsequent neurovascular exploration, reconstructive surgical procedures, and left tibia fracture decompression and fixation on the first admission day. On the seventh day, fever with a peak of $39.0^{\circ} \mathrm{C}$ and a hematoma in the left elbow and forearm with severe exudation emerged. The leukocyte count increased to $11.18 \times 10^{9} / \mathrm{L}$ with an elevated neutrophil proportion (88.8\%). Mild abnormal hepatic dysfunction was noticed; alanine aminotransferase was $82 \mathrm{IU} / \mathrm{L}$ (reference range $<50 \mathrm{IU} / \mathrm{L}$ ), and aspartate aminotransferase was $83 \mathrm{IU} / \mathrm{L}$ (reference range $<40 \mathrm{IU} / \mathrm{L}$ ). Prior to incision and drainage, peripheral blood samples were sent to the clinical microbiology lab for culturing. Ceftazidime was given empirically and intravenously as $2000 \mathrm{mg}$ every $12 \mathrm{~h}$. The blood culture reported a positive result after $19 \mathrm{~h}$ of incubation, and this strain, designated as WCHRI065437, was then identified as $R$. mannitolilytica by the VITEK 2 Compact system (strain name WCHRI065437). The antimicrobial therapy was adjusted to ceftazidime $2000 \mathrm{mg}$ every $8 \mathrm{~h}$ for 2 weeks. Finally, the patient completely recovered and was discharged.

\section{Clinical information of case 2}

A 35-year-old male who had received a biopsy and chemotherapy in the past was admitted for surgery due to left femur osteosarcoma. The subsequent operations involved resection of a distal femoral tumor, assembled tumor knee replacement, neurovascular exploration and muscle reconstruction. A fever peaking at $38.7^{\circ} \mathrm{C}$ without a focal infection was noticed on the fourth day. Blood tests revealed WBC $4.45 \times 10^{9} / \mathrm{L}$ (neutrophil $87 \%$ ), elevated C-reactive protein of 12.20 $\mathrm{mg} / \mathrm{L}$ and IL-6 of $12.37 \mathrm{pg} / \mathrm{mL}$. Alanine aminotransferase was mildly elevated to $97 \mathrm{IU} / \mathrm{L}$ (reference range $<50 \mathrm{IU} / \mathrm{L}$ ), and aspartate aminotransferase was mildly elevated to $75 \mathrm{IU} / \mathrm{L}$ (reference range $<40 \mathrm{IU} / \mathrm{L}$ ). Empirical intravenous antibiotic therapy with ciprofloxacin $400 \mathrm{mg}$ per $12 \mathrm{~h}$ was immediately initiated. Peripheral blood culture revealed a bacterium designated as WCHRI05162 after $26 \mathrm{~h}$ of incubation that was also identified as $R$. mannitolilytica by the VITEK 2 Compact system. However, the leukocyte count kept climbing to $13.9 \times 10^{9} / \mathrm{L}$ despite 3 days' treatment. Since additional disc diffusion tests showed that the bacterium was susceptible to cefoperazone/sulbactam (38 mm), cefoperazone/sulbactam $1.5 \mathrm{~g}$ every $12 \mathrm{~h}$ was added. The patient became completely afebrile 2 days later and was transferred to a rehabilitation hospital.

\section{Isolates identification}

Medium sized, round shaped, moist raised light brown colonies with neat edges were observed. Although the two isolates were misidentified as $R$. mannitolilytica by the VITEK 2 Compact system, they were accurately identified as $R$. insidiosa by both MALDI-TOF MS and $16 \mathrm{~S}$ rRNA gene sequencing. Using either the universal primers $27 \mathrm{~F} / 1492 \mathrm{R}$ or the species-specific primers Rp-F1/R38R1, the nucleotide identity was $99 \%$.

\section{MIC determination}

Both strains were resistant to amikacin, amoxicillin/clavulanate, ampicillin, aztreonam, gentamicin, polymyxin $B$, tobramycin, and nitrofurantoin, but susceptible to ciprofloxacin, levofloxacin, ceftriaxone, piperacillin/tazobactam, tigecycline, and trimethoprim/sulfamethoxazole. Isolate WCHRI065162 from case 2 was resistant to imipenem and meropenem and was intermediate to cefepime and ceftazidime, whereas isolate WCHRI065437 was susceptible to all of them (Table 1).

\section{Whole genome sequencing, species confirmation, and phylogenetics}

The genome sizes revealed by the draft genomes of $R$. insidiosa strains WCHRI065162 and WCHRI065437 were $5,923,114 \mathrm{bp}$ and $6,113,916 \mathrm{bp}$, respectively. Their $\mathrm{G}+\mathrm{C}$ contents were 63.41 and $63.63 \%$, respectively (Table 3). To confirm the results of rough species identification based on individual gene sequencing, ANI calculations were performed between two isolates and type strains of $R$. picketti and $R$. solanacearum. The results were interpreted according to the suggested threshold [13], showing that neither strain belonged to these two species. However, lacking genomic data of type strains of species $R$. insidiosa, $R$. mannitolilytica, $R$. pseudosolanacearum and $R$. syzygii in the public database, a phylogenetic analysis was performed on a total of 13 strains, including nine non-type strains of these four species, two publicly available type strains, and two from this study, to determine the closest related species of strains WCHRI065162 and WCHRI065437. Having determined that both isolates clustered with species $R$. insidiosa with high bootstrap values on the phylogenetic tree (Fig. 1), we observed that none of results from pairwise ANI calculations between the two isolates and all other genomes exceeded 95\%, except for those calculated with $R$. insidiosa ATCC 49129 and FC1138 (Table 4). The results from the BLASTn algorithm of previous amplicon sequencing confirmed that WCHRI065162 and WCHRI065437 were strains of the species $R$. insidiosa. There were 74,392 bp SNPs between our two isolates, suggesting they were not the same clone. The circular map (Fig. 2) showed that several fragments were absent 
Table 3 Genome information of two $R$. insidiosa isolates in this study

\begin{tabular}{lll}
\hline Isolate & $\begin{array}{l}\text { R. insidiosa } \\
\text { WCHRI065162 }\end{array}$ & $\begin{array}{l}\text { R. insidiosa } \\
\text { WCHRI065437 }\end{array}$ \\
\hline total contig & 53 & 47 \\
total sequence (bp) & $5,925,661$ & $6,116,439$ \\
N50(bp) & 329,971 & 910,997 \\
G + C\% & 63.41 & 63.63 \\
coding DNA sequence & 5549 & 5717 \\
Largest contig (bp) & 602,553 & $1,542,049$ \\
CDS & 5549 & 5717 \\
tRNAs & 54 & 55 \\
rRNAs & 3 & 3 \\
tmRNA & 1 & 1 \\
class D $\beta$-lactamase gene & bla OXA-569、bla bXA-570 & bla OXA-573、bla OXA-574 \\
GenBank accession No. & NZ_PKPC00000000.1 & NZ_PKPA00000000.1 \\
\hline
\end{tabular}

in our isolates compared to the chromosome sequences of $R$. insidiosa ATCC 49129 and FC1138.

We identified multiple putative virulence factors, including those involved in quorum sensing, biofilm formation, the production of bacteriocins, adhesin and invasins based on genome annotation. Both isolates contained seven quorum sensing and biofilm formation genes (pelA, pelB, pelC, pelD, pelE, pelF, and pelG) involved in glucose-rich biofilm formation; these were slightly different between the two isolates due to amino acid substitutions ( 3 in PelA, 11 in PelB, 2 in PelC, and 4 in PelD). Adhesion genes related to autotransporter adhesin, sigma-fimbriae tip adhesin, fimbrial adhesin and type $\mathrm{V}$ secretory pathway adhesin AidA were found in both isolates. Secretion systems related to adhesin, biofilm formation, and pilus assembly were also found. WCHRI065162 contained T2SS, T4SS, T5SS, T6SS and
T7SS (the type II, IV, V, VI, VII secretion systems), while WCHRI065437 only contained T2SS, T4SS, T6SS and T7SS (the type II, IV, VI, VII secretion systems). Compared to $R$. insidiosa ATCC 49129 and FC1138, our isolates did not have the $h c p$ gene. Both isolates harbored genes related to colicin $\mathrm{V}$ and the bacteriocin production cluster.

WCHRI065437 carried two novel variants of the gene encoding class D $\beta$-lactamase ( $b l a_{\text {OXA-573 }}, b l a_{\text {OXA-574, }}$, accession numbers MG736320 and MG736321, respectively) which we deposited into the Bacterial Antimicrobial Resistance Reference Gene Database (https://www.ncbi.nlm.nih.gov/bioproject/PRJNA313047). WCHRI065162 carried two genes encoding class D $\beta$-lactamase (bla $a_{\mathrm{OXA}-569}$ and bla $a_{\mathrm{OXA}-570}$, accession numbers MG736316 and MG736317). OXA-569 and OXA-574 were highly similar to OXA-22 (amino acid identity 85 and $86 \%$, respectively). The proteins OXA-570 and OXA-573 were also highly similar to OXA-60 (amino acid identity 84 and $86 \%$, respectively). The promoter of $b l a_{\text {OXA-573 }}$ was $46 \mathrm{bp}$ shorter than that of $b l a_{\text {OXA-570. All of }}$ these $b l a_{\mathrm{OXA}}$ alleles were located on the chromosomes without transposable elements nearby, suggesting that they are intrinsic in $R$. insidiosa. None of the known plasmid replicons were found in either of the two isolates.

\section{Real-time RT-PCR and cloning experiments to explore the related class $D \beta$-lactamase-encoding genes}

There was no significant difference between $b l a_{\text {OXA-570 }}$ copy numbers in $R$. insidiosa WCHRI065162 and bla $a_{\text {OXA-573 }}$ copy numbers in $R$. insidiosa WCHRI065437 (fold difference $=0.79$ ), suggesting difference in gene expression at the mRNA level was not responsible for the carbapenem-resistant phenotype. However, the MICs of recombinant harboring plasmids $\mathrm{pBC} 1$ carrying $b l a_{\mathrm{OXA}-570}$ against carbapenem and other $\beta$-lactams were significantly higher than those of reference strain E. coli DH10B (Table 5). There were no significant differences in

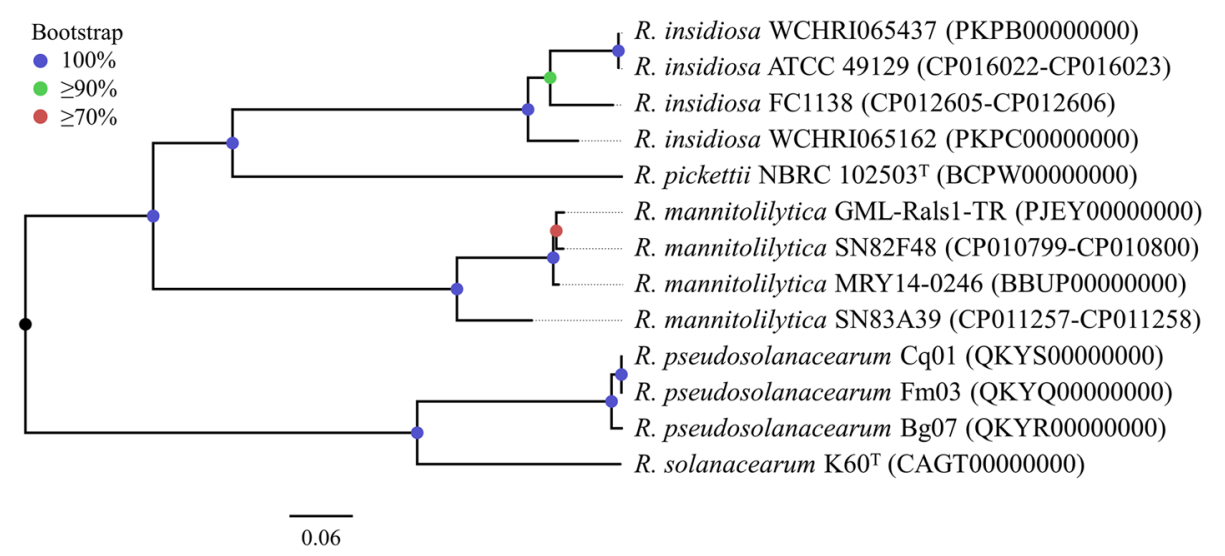

Fig. 1 Phylogenetic tree based on core genome SNP. The tree was inferred using the maximum likelihood method under the GTRGAMMA model with a 1000-bootstrap test. Support values are colored based on confidence at internal nodes 
Table 4 Calculated average nucleotide identity (ANI) between WCHRI065162, WCHRI065437 and other published strains of genus Ralstonia

\begin{tabular}{lll}
\hline Strains & R. insidiosa WCHRI065162 (\%) & R. insidiosa WCHRI065437 (\%) \\
\hline R. insidiosa WCHRI065162 & - & 96.70 \\
R. insidiosa WCHRI065437 & 96.70 & - \\
R. insidiosa FC1138 & 96.65 & 97.09 \\
R. insidiosa ATCC 49129 & 96.50 & 99.67 \\
R. pickettii NBRC 102503 ${ }^{\top}$ & 85.98 & 85.93 \\
R. mannitolilytica SN82F48 & 85.86 & 85.70 \\
R. mannitolilytica SN83A39 & 85.70 & 85.66 \\
R. mannitolilytica MRY14-0246 & 85.62 & 85.88 \\
R. mannitolilytica GML-Rals1-TR & 85.48 & 85.91 \\
R. solanacearum K60' & 84.33 & 84.19 \\
R. pseudosolanacearum Fm03 & 83.66 & 83.62 \\
R. pseudosolanacearum Bg07 & 83.65 & 83.64 \\
R. pseudosolanacearum Cq01 & 83.64 & 83.61
\end{tabular}

antimicrobial susceptibility against cefepime, ceftazidime, imipenem or meropenem between E. coli DH10B and other recombinants harboring plasmids $\mathrm{pBC} 2$ (bla $\left.a_{\text {OXA-570 }}\right)$, pBC3 (bla $\left.a_{\text {OXA-569 }}\right)$, pBC4 $\left(b l a_{\text {OXA-574 }}\right)$ (Table 5). This suggested that in our strains only OXA-570 showed carbapenem hydrolase activity, which may be responsible for the carbapenem-resistance of isolate WCHRI065162.

\section{Discussion}

Few cases of infections caused by $R$. insidiosa have been reported due to the limited awareness of the pathogen.

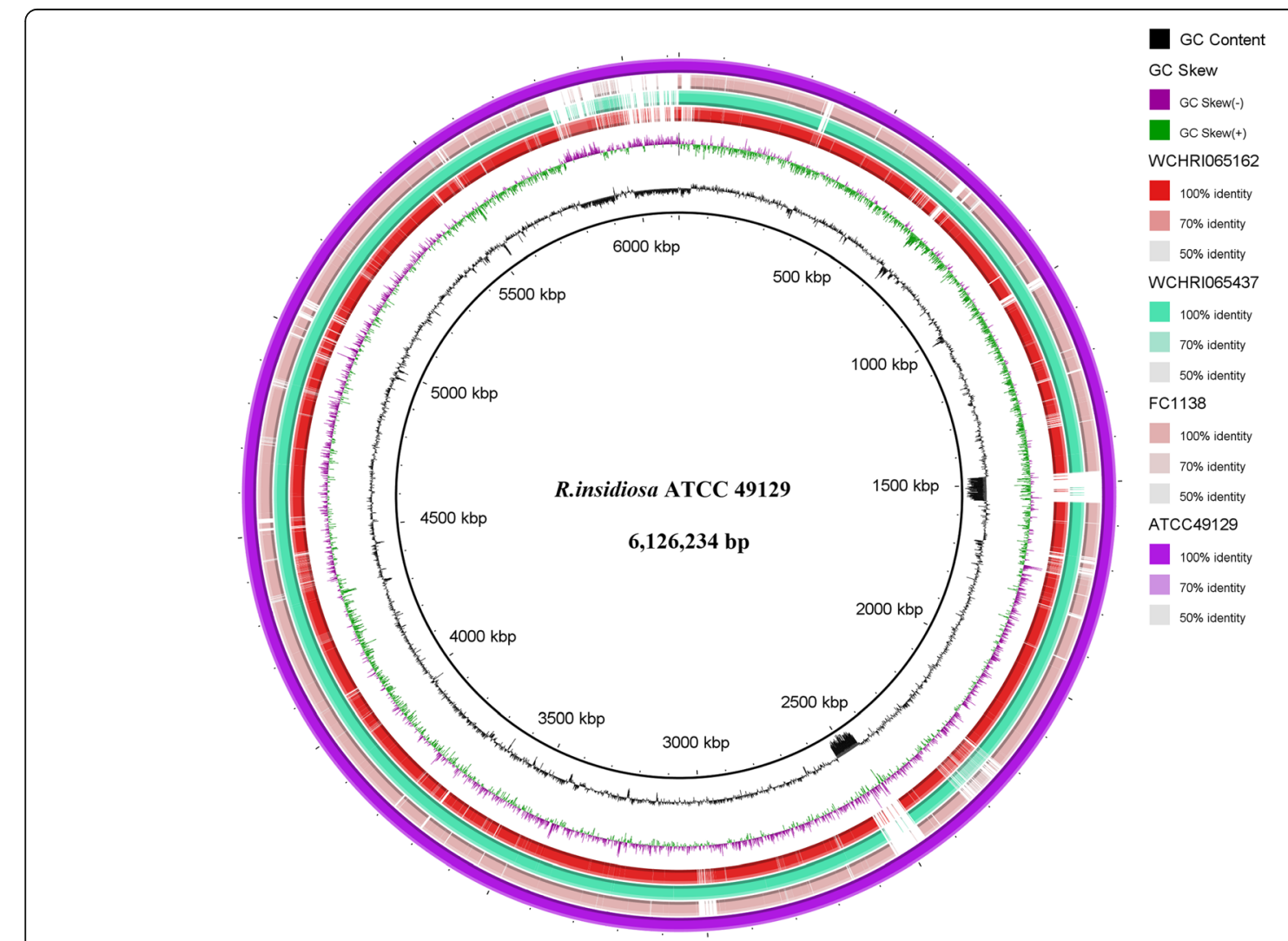

Fig. 2 Comparative analysis among R. insidiosa genomes of our isolates and previously published strains ATCC 49129 and FC1138 
Table 5 MICs to $\beta$-lactam antibiotics of E. coli DH1OB and recombinant harboring plasmids $\mathrm{pBC1}, \mathrm{pBC2}, \mathrm{pBC} 3$ and $\mathrm{pBC} 4$

\begin{tabular}{llllll}
\hline B-lactam & E.coli & & & & \\
\cline { 2 - 6 } Antibiotics & $\mathrm{DH10B}$ & $\mathrm{pBC1}$ & $\mathrm{pBC2}$ & $\mathrm{pBC} 3$ & $\mathrm{pBC}$ \\
\hline Meropenom & 0.03 & 8 & 0.03 & 0.03 & 0.03 \\
Imipenem & 0.5 & 2 & 0.5 & 0.5 & 0.5 \\
Cefepime & $<0.03$ & 0.06 & $<0.03$ & $<0.03$ & $<0.03$ \\
Cefoxitin & 1 & 8 & 4 & 4 & 4 \\
Ceftazidime & $<0.125$ & 0.5 & $<0.125$ & $<0.125$ & $<0.125$ \\
Ampicillin & 8 & 256 & 8 & 16 & 16 \\
Amoxicillin & 4 & 128 & 4 & 16 & 16 \\
\hline
\end{tabular}

pBC1: plasmid pBC-SK carrying bla $a_{\mathrm{OXA}-570 ;}$ pBC2: plasmid pBC-SK carrying bla $a_{\mathrm{OXA}-573 ;}$ pBC3: plasmid pBC-SK carrying bla $a_{\mathrm{OXA}-569 ;}$ pBC4: plasmid $\mathrm{pBC}-\mathrm{SK}$ carrying bla $a_{\mathrm{OXA}-574}$

Ralstonia spp. are emerging as global opportunistic pathogens affecting immunocompromised patients. Even without person-to-person transmission, the prevalence of Ralstonia infection is notably increasing. The bacteria reproduce in wet conditions and can survive in harsh environments (even in weak disinfectants) for long periods. The species exist widely in external aqueous environments including municipal water and medical water purification systems $[2,14]$. As the bacteria can pass through $0.2-\mu \mathrm{m}$ filters during the sterilization process, medical products may be contaminated during the manufacturing phase [15]. The species can create biofilms on the surfaces of medical supplies and produce toxins. Many infectious cases caused by $R$. picketti and $R$. mannitolilytica are due to the use of contaminated solutions, blood products, chlorhexidine, saline solution, and sterile water as well as the colonization of medical devices (tap water and water used for hemodialysis, bronchoscope flushing, and heparin for flushing) [16-18].

We described two rare nosocomial cases of bloodstream infections caused by $R$. insidiosa, although we could not track their source. Both of our patients were at a high risk for infection. One patient had a malignant tumor of the femur and underwent chemotherapy and surgery, while the other patient was bedridden for a long time because of severe multiple fractures and several surgical operations. High risk factors listed in the literature are cancer, blood vessel catheters, chronic renal failure, ischemic heart disease, newborn and other immunocompromised conditions [1]. The common symptom in these two cases was obvious fever, while the common abnormal routine test results were increased neutrophils and aminotransferase.

Based on our results, we recommend MALDI-TOF MS for rapid identification of $R$. insidiosa. Due to the inaccurate biochemical reactions, both of our $R$. insidiosa isolates were misidentified as $R$. mannitolilytica by the VITEK 2 Compact system, a widely accepted commercial automated biochemical identification instrument. The manual identification keys are that $R$. insidiosa metabolizes nitrate but not mannitol or arabinose, $R$. picketti metabolizes nitrate and arabinose but not mannitol, and $R$. mannitolilytica metabolizes only mannitol but not nitrate or arabinose [3]. Ralstonia spp. are very close to the Burkholderia cepacia complex and Pseudomonas fluorescens; the previous name of $R$. picketti was Burkholderia pickettii. Previously, we reported that the VITEK 2 system misidentified Burkholderia pseudomallei as Burkholderia cepacia [19]. This reminds us that clinicians should be cautious with automated identification of non-fermentative gram-negative bacilli, especially Burkholderia reported by the VITEK 2 system. For species identification, our study showed that using either the universal primers $27 \mathrm{~F} / 1492 \mathrm{R}$ or the species-specific primers Rp-F1/R38R1, 16S rRNA gene sequencing were practical. Additionally, species-specific primers Rp-F1/R38R1 were much more rapid for identifying $R$. insidiosa from $R$. picketti and $R$. mannitolilytica, because no amplicon band of $R$. picketti or $R$. mannitolilytica could be observed on the electrophoresis gel. However, considering turnaround time and PCR identification cost, MALDI-TOF MS would be a better choice. Our results supported the general consensus that MALDI-TOF MS could provide rapid and accurate results [20].

Both of our $R$. insidiosa isolates were multi-drug resistant, even the strain that was carbapenems-resistant. The antibiotic susceptibility data for $R$. insidiosa are very limited, not to mention that of clinical isolates. A study reporting antibiotic susceptibility profiles of 15 environmental $R$. insidiosa isolates showed that quinolones and the folate pathway inhibitors (sulfamethoxazole/trimethoprim) were most effective [21]. MICs results of our clinical isolates were consistent with those results and revealed that piperacillin/tazobactam and tigecycline were also susceptible alternatives. Considering accessibility, cost and side effects, quinolones may be a good choice for initial empirical therapy. However, since antimicrobial susceptibility (i.e., carbapenem susceptibility) discriminated between isolates, we still recommend that clinicians select antibiotics according to their MICs results.

Genome sequencing of our $R$. insidiosa WCHRI065162 and WCHRI065437 has improved current understanding of the antibiotic resistance and pathogenicity of this species. Due to a wide range of SNPs, our isolates were not the same clone, and neither of our isolates was close to those two fully assembled genomes of $R$. insidiosa in the NCBI database. Bacterial secretion systems present on the cell membranes are the cellular components used by pathogenic bacteria to secrete their virulence factors in order to invade the host cells. Although the classification is not very clear or complete, pathogenic gram-negative bacteria possess at least six secretory systems related to virulence factors [22]. The T2SS (Type II secretion system) contains a pseudopilus and secretes toxins and 
hydrolyzing enzymes. A main role of T4SS (type IV secretion system) is to facilitate the spread of drug-resistant genes located on plasmids through conjunction. T5SS (type V secretion system) is mainly involved in virulence factor secretion, intercellular adhesion and biofilm formation. The T3SS (type III secretion system), especially type IV pilus (T4P) biogenesis system, and T6SS (type VI secretion system) are now recognized as pathogenicity hallmarks in many gram-negative bacteria [23, 24]. These systems deliver bacterial proteins called effectors into neighboring bacteria or host cells, leading to cytotoxicity and cell death of targets. Both R. insidiosa ATCC 49129 and $R$. insidiosa FC1138 have T3SS and T6SS gene clusters, but our two isolates carried only T6SS gene clusters, implying that T6SS may be more crucial for the virulence of $R$. insidiosa. T7SS (type VII secretion system), rarely found in gram-negative bacteria, is a specialized secretion apparatus required for the virulence of mycobacteria [22]. However, all of $R$. insidiosa ATCC 49129, FC1138 and our isolates have T7SS. Further fundamental research is needed to reveal the role of T7SS in the virulence of $R$. insidiosa.

Quorum sensing, an efficient bacterial cell-to-cell communication process for population growth, involves bioluminescence, secretion of virulence factors, production of public products, and biofilm formation [25]. Both of our isolates contained biofilm formation genes (Pel) related to the generation of glucose-rich extracellular polysaccharide (PEL) and the construction of biofilm matrix. To some extent, these genes may be responsible for pathogenicity and multi-drug resistance phenotypes of $R$. insidiosa. Bacteriocin, one family of microbial defense systems, is now considered to be the most diverse and naturally abundant antimicrobial molecule [26]. Colicin V and bacteriocin production cluster subsystems were found in both of our isolates.

Both of our isolates were resistant to the $\beta$-lactam antibiotics, polymyxin $\mathrm{B}$, and aminoglycosides. It is known that two chromosomally encoded class $D \beta$-lactamases, OXA-22 and OXA-60, are related to multidrug-resistance of Ralstonia spp. [27, 28]. OXA-22 is active against benzylpenicillin, cloxacillin and some cephalosporins, while OXA-60 is active against imipenem. Also, bla $a_{\text {OXA-569, }}$ $b l a_{\mathrm{OXA}-570}, b l a_{\mathrm{XA}-573}$ and $b l a_{\mathrm{OXA}-574}$ are very similar to $b l a_{\mathrm{OXA}-22}$ and $b l a_{\mathrm{OXA}-60}$. Although both our strains harbored a $b l a_{\mathrm{OXA}-60}$ variant $\left(b l a_{\mathrm{OXA}-570}\right.$ and $\left.b l a_{\mathrm{OXA}-573}\right)$, only isolate WCHRI065162 carrying $b l a_{\text {OXA-570 }}$ was resistant to carbapenems. The cloning experiment showed that variant OXA-573 revealed in this study had no carbapenem hydrolase activity. Polymyxin, a cationic polypeptide interacting with the negatively charged phosphate groups in the lipopolysaccharide of the bacterial outer membrane, is the last resort antibiotic against extensively drug-resistant gram-negative bacillus infection. Many pathogens, including Burkholderia spp., are inherently resistant to antimicrobial peptides [29]. Members of the $R$. pickettii lineage, including $R$. insidiosa, are intrinsically resistant to colistin [30], which clinicians should be aware of prior to issuing antibiotic prescriptions.

The main limitation of this study is that the source of bloodstream infection in our patients was unknown. Since Ralstonia exists widely in the external aqueous environment, a contaminated water supply or parenteral fluid could be the primary source of infection. However, no contamination in our hospital water supply was found during follow up surveillance. Due to our previous limited understanding of Ralstonia, no sample from environment at that time was reserved for further investigation. This report serves as an alert for medical workers and researchers to pay more attention to infection caused by $R$. insidiosa.

In conclusion, we described two cases of nosocomial bloodstream infection and the microbiological profile of their causable $R$. insidiosa strains. MALDI-TOF MS was cost-effective for rapid species identification. Clinicians should be aware that species $R$. insidiosa is capable of being resistant to many routinely used antibiotics, even carbapenems.

\section{Abbreviations}

ANI: Average nucleotide identity; CLSI: Clinical and Laboratory Standards Institute; MALDI-TOF MS: Matrix assisted laser desorption ionization-time of flight mass spectrometry; MIC: Minimum inhibitory concentrations;

SNP: Single nucleotide polymorphism; SS: Secretion system

\section{Acknowledgments}

We are grateful to Xiaoxia Zhang for providing technical support.

\section{Funding}

This study was supported by the Technology Support Program of Sichuan Provincial Science and Technology Agency, China (project Nos. 2015SZ02344 and $2018 \mathrm{HH} 0031$ ). The funders had no role in the design of the study and collection, analysis, and interpretation of data or in writing the manuscript.

\section{Availability of data and materials}

The data used for this study are available upon request. Please contact our corresponding author.

\section{Authors' contributions}

Concept and design: X.W.; Acquisition of data: Q.F., Y.F., and X.W.; Analysis/ Interpretation of data: Q.F., Y.F., P.F., X.W. and Z.Z.; Drafting of manuscript: Q.F., and X.W. All authors have read and approved the manuscript.

\section{Ethics approval and consent to participate}

This study was approved by the Ethics Committee of West China Hospital, Sichuan University with a waiver of consent because the medical records and bacterial isolates used in this study were obtained from previous routine clinical activities. The committee deemed that exempting informed consent would not adversely affect the rights or health of the patients.

\section{Consent for publication}

Written informed consent has been acquired from the patients for the publication of this manuscript and all information contained herein.

Competing interests

The authors declare that they have no competing interests. 


\section{Publisher's Note}

Springer Nature remains neutral with regard to jurisdictional claims in published maps and institutional affiliations.

\section{Author details}

${ }^{1}$ Center of Infectious Diseases, West China Hospital, Sichuan University, Chengdu, China. ${ }^{2}$ Division of Infectious Diseases, State Key Laboratory of Biotherapy, Chengdu, China. ${ }^{3}$ Department of Infection Control, West China Hospital, Sichuan University, Chengdu, China.

Received: 17 November 2018 Accepted: 12 April 2019

Published online: 23 April 2019

\section{References}

1. Ryan MP, Adley CC. Ralstonia spp.: emerging global opportunistic pathogens. Eur J Clin Microbiol Infect Dis. 2014;33:291-304.

2. Chen YY, Huang WT, Chen CP, Sun SM, Kuo FM, Chan YJ, et al. An outbreak of Ralstonia pickettii bloodstream infection associated with an intrinsically contaminated normal saline solution. Infect Control Hosp Epidemiol. 2017; 38:444-8.

3. Coenye T, Goris J, De Vos P, Vandamme P, LiPuma JJ. Classification of Ralstonia pickettii-like isolates from the environment and clinical samples as Ralstonia insidiosa sp. nov. Int J Syst Evol Microbiol. 2003;53:1075-80.

4. Ryan MP, Pembroke JT, Adley CC. Genotypic and phenotypic diversity of Ralstonia pickettii and Ralstonia insidiosa isolates from clinical and environmental sources including high-purity water. Diversity in Ralstonia pickettii. BMC Microbiol. 2011;11:194.

5. CLSI. Performance Standards for Antimicrobial Susceptibility Testing. 28th ed. CLSI supplement M100. Pennsylvania: Clinical and Laboratory Standards Institute; 2017

6. Bolger AM, Lohse M, Usadel B. Trimmomatic: a flexible trimmer for Illumina sequence data. Bioinformatics. 2014;30:2114-20.

7. Bankevich A, Nurk S, Antipov D, Gurevich AA, Dvorkin M, Kulikov AS, et al. SPAdes: a new genome assembly algorithm and its applications to singlecell sequencing. J Comput Biol. 2012;19:455-77.

8. Page AJ, Cummins CA, Hunt M, Wong VK, Reuter S, Holden MT, et al. Roary: rapid large-scale prokaryote pan genome analysis. Bioinformatics. 2015;31: 3691-3.

9. Stamatakis A. RAxML version 8: a tool for phylogenetic analysis and postanalysis of large phylogenies. Bioinformatics. 2014;30:1312-3.

10. Alikhan NF, Petty NK, Ben Zakour NL, Beatson SA. BLAST ring image generator (BRIG): simple prokaryote genome comparisons. BMC Genomics. 2011;12:402

11. Livak KJ, Schmittgen TD. Analysis of relative gene expression data using real-time quantitative PCR and the 2(-Delta Delta C(T)) method. Methods (San Diego, Calif). 2001;25(4):402-8.

12. Michael Green, Joseph Sambrook. Molecular Cloning: A Laboratory Manual, Fourth Edition. Cold Spring Harbor Laboratory Press, Cold Spring Harbor, NE 2012.

13. Chun J, Oren A, Ventosa A, Christensen H, Arahal DR, da Costa MS, et al. Proposed minimal standards for the use of genome data for the taxonomy of prokaryotes. Int J Syst Evol Microbiol. 2018;68:461-6.

14. Vincenti S, Quaranta G, De Meo C, Bruno S, Ficarra MG, Carovillano S, et al. Non-fermentative gram-negative bacteria in hospital tap water and water used for haemodialysis and bronchoscope flushing: prevalence and distribution of antibiotic resistant strains. Sci Total Environ. 2014;499:47-54.

15. Anderson RL, Bland LA, Favero MS, McNeil MM, Davis BJ, Mackel DC, et al. Factors associated with Pseudomonas pickettii intrinsic contamination of commercial respiratory therapy solutions marketed as sterile. Appl Environ Microbiol. 1985;50:1343-8.

16. Tejera D, Limongi G, Bertullo M, Cancela M. Ralstonia pickettii bacteremia in hemodialysis patients: a report of two cases. Rev Bras Ter Intensiva. 2016;28: 195-8. https://doi.org/10.5935/0103-507X.20160033.

17. Lai HW, Shen YH, Chien LJ, Tseng SH, Mu JJ, Chan YJ, et al. Outbreak of Ralstonia pickettii bacteremia caused by contaminated saline solution in Taiwan. Am J Infect Control. 2016:44:1191-2.

18. Lucarelli C, Di Domenico EG, Toma L, Bracco D, Prignano G, Fortunati M, et al. Ralstonia mannitolilytica infections in an oncologic day ward: description of a cluster among high-risk patients. Antimicrob Resist Infect Control. 2017; 6:20. https://doi.org/10.1186/s13756-017-0178-z.
19. Zong Z, Wang X, Deng Y, Zhou T. Misidentification of Burkholderia pseudomallei as Burkholderia cepacia by the VITEK 2 system. J Med Microbiol. 2012;61:1483-4.

20. Mellmann A, Cloud J, Maier T, Keckevoet U, Ramminger I, Iwen P, et al. Evaluation of matrix-assisted laser desorption ionization-time-of-flight mass spectrometry in comparison to 165 rRNA gene sequencing for species identification of nonfermenting bacteria. J Clin Microbiol. 2008;46:1946-54.

21. Ryan MP, Adley CC. The antibiotic susceptibility of water-based bacteria Ralstonia pickettii and Ralstonia insidiosa. J Med Microbiol. 2013;62:1025-31.

22. Costa TR, Felisberto-Rodrigues C, Meir A, Prevost MS, Redzej A, Trokter M, et al. Secretion systems in gram-negative bacteria: structural and mechanistic insights. Nat Rev Microbiol. 2015;13(6):343-59.

23. Russell $A B$, Peterson SB, Mougous JD. Type VI secretion system effectors: poisons with a purpose. Nat Rev Microbiol. 2014;12:137-48.

24. Cunnac S, Boucher C, Genin S. Characterization of the cis-acting regulatory element controlling HrpB-mediated activation of the type III secretion system and effector genes in Ralstonia solanacearum. J Bacteriol. 2004;186: 2309-18.

25. Solano C, Echeverz M, Lasa I. Biofilm dispersion and quorum sensing. Curr Opin Microbiol. 2014;18:96-104.

26. Riley MA, Wertz JE. Bacteriocins: evolution, ecology, and application. Annu Rev Microbiol. 2002;56:117-37.

27. Suzuki M, Nishio H, Asagoe K, Kida K, Suzuki S, Matsui M, et al. Genome sequence of a Carbapenem-resistant strain of Ralstonia mannitolilytica. Genome Announc. 2015;3. https://doi.org/10.1128/genomeA.00405-15.

28. Girlich D, Naas T, Nordmann P. OXA-60, a chromosomal, inducible, and imipenem-hydrolyzing class D beta-lactamase from Ralstonia pickettii. Antimicrob Agents Chemother. 2004;48:4217-25.

29. Loutet SA, Valvano MA. Extreme antimicrobial peptide and polymyxin B resistance in the genus burkholderia. Front Microbiol. 2011;2:159. https://doi. org/10.3389/fmicb.2011.00159.

30. Vaneechoutte M, Kampfer P, De Baere T, Falsen E, Verschraegen G. Wautersia gen. nov., a novel genus accommodating the phylogenetic lineage including Ralstonia eutropha and related species, and proposal of Ralstonia [Pseudomonas] syzygii (Roberts et al. 1990) comb. nov. Int J Syst Evol Microbiol. 2004;54:317-27.

Ready to submit your research? Choose BMC and benefit from:

- fast, convenient online submission

- thorough peer review by experienced researchers in your field

- rapid publication on acceptance

- support for research data, including large and complex data types

- gold Open Access which fosters wider collaboration and increased citations

- maximum visibility for your research: over $100 \mathrm{M}$ website views per year

At BMC, research is always in progress.

Learn more biomedcentral.com/submissions 